\title{
Indicateurs sur la ressource en eau estimés par une modélisation pluie-débit régionalisée : la base de données Web LoiEau
}

\author{
Nathalie Folton ${ }^{*}$ et Patrick Arnaud \\ INRAE, Aix Marseille Univ., RECOVER, Aix-en-Provence, France
}

Reçu le 23 mars 2020 / Accepté le 10 juillet 2020

\begin{abstract}
Résumé - Une approche conceptuelle parcimonieuse a été développée pour la quantification et la gestion de la ressource en eau sur des territoires dépourvus d'information. La base de données Web LoiEau présentée dans cet article, constitue le résultat de l'application de cette méthode à l'exutoire de plus de 130000 bassins versants non instrumentés sur le territoire national. L'approche régionale exploite une information hydrométéorologique récente et de bonne qualité la plus exhaustive possible (données climatiques de la réanalyse SAFRAN et données hydrologiques de la banque Hydro) et résulte d'avancées méthodologiques réalisées sur la détermination d'une structure bien optimisée d'un modèle hydrologique journalier adapté à une gamme de fonctionnements hydrologiques variés, contraint seulement par deux paramètres pour permettre sa régionalisation et son utilisation sur des bassins non jaugés. La base de données Web LoiEau fournit des chroniques hydrologiques simulées de 1958 à 2018 au pas de temps journalier, à partir desquelles de multiples indicateurs hydrologiques sont extraits, permettant de caractériser la ressource en eau dans son ensemble (étiage, saisonnalité, bilan). Cette base est diffusée pour les services de l'État via une interface Web. L'étude des incertitudes liées à l'échantillonnage des données observées a permis d'établir des intervalles de confiance pour chaque indicateur hydrologique. Un indice de confiance en la méthode est aussi proposé pour chaque bassin afin de qualifier le degré d'applicabilité des résultats dans le cas où les spécificités locales les rendent inappropriés.
\end{abstract}

Mots clés : modélisation pluie-débit / méthode régionale / indice d'étiage / base de données sur l'eau

\begin{abstract}
Water resource indicators estimated by regionalized daily rainfall-runoff model: LoiEau Web Database. A parsimonious conceptual approach has been developed for the quantification and management of water resources in territories without information. The LoiEau Web database, presented in this article, is the result of applying this method to the outlet of more than 130000 ungauged catchments over the French territory. The regional approach exploits recent and good quality hydro-meteorological information that is as exhaustive as possible (the SAFRAN reanalysis and the Hydro database) and is the result of methodological advances made in determining a well-optimized structure of a daily hydrological model adapted to a varied range of hydrological process, constrained by only two parameters to allow its regionalization and use on ungauged basins. The LoiEau Web database provides simulated hydrological data from 1958 to 2018 at daily time steps, from which multiple hydrological indicators are extracted to characterize water resources (low flow, seasonality, mean annual streamflow). This database is distributed via a Web interface. Uncertainties were calculated by resampling of the observed data and allowed to calculate confidence intervals for each hydrological index on each catchment. A confidence index in the method is also proposed for each catchment in order to qualify the degree of applicability of results in the event that local specificities make them inappropriate.
\end{abstract}

Keywords: regionalized daily rainfall-runoff model / low-flow indices / hydrological database

\footnotetext{
*Correspondance : nathalie.folton@inrae.fr
} 


\section{Introduction}

Les réseaux de mesures hydro-météorologiques ne sont pas assez denses par rapport à la variabilité des processus pour espérer s'appuyer uniquement sur des observations locales pour étudier les phénomènes hydrologiques en tout point du territoire. Le problème de la connaissance des débits sur les sites non jaugés est une question clé en hydrologie appliquée. Par exemple dans le contexte de la Directive Cadre européenne sur l'Eau (DCE), le régime hydrologique doit être pris en compte pour évaluer les masses d'eau sur l'ensemble du réseau hydrographique. Certains débits sont notamment utilisés par les gestionnaires de l'eau pour appliquer le code de l'environnement. L'application de la réglementation nécessite donc d'avoir une estimation des débits en n'importe quel point du réseau hydrographique. Cette question a été l'un des grands axes de recherche de la décennie PUB (Prediction in Ungauged Basin) lancé par l'IAHS en 2003, et du programme de recherche international FRIEND (Flow Regimes from International Experimental and Network Data). Une synthèse exhaustive a été publiée sur les méthodes de modélisation en contexte non jaugé permettant d'estimer différentes signatures hydrologiques, parmi lesquelles les indices d'étiage (Blöschl et al., 2013). La branche européenne du programme FRIEND (EURO FRIENDWater Low Flow group), sous l'égide de l'UNESCO s'est orientée ensuite vers la problématique de détermination des indices d'étiages et leur régionalisation (Laaha et al., 2013b). Le rapport PUB soulignait que les méthodes basées sur les processus étaient sous-représentées dans la littérature. En effet les approches statistiques sont plus classiquement utilisées pour l'estimation des indices d'étiage (Laaha et al., 2013a). Mais ces méthodes ne peuvent traiter qu'un indice à la fois et ne sont pas adaptées pour évaluer l'impact de changements environnementaux ou climatiques sur ces indices. La modélisation des écoulements à partir de la pluie est une alternative intéressante car elle permet de remédier à ces deux inconvénients et elle présente en plus l'avantage de prendre en compte la dynamique de l'hydrogramme et des différents processus liés à l'étiage. La modélisation hydrologique permet aussi d'être indépendante des indices d'étiage souhaités et de garder une cohérence dans leur estimation lorsqu'on souhaite en estimer plusieurs. C'est dans ce contexte que la méthode LoiEau a été développée et améliorée au cours de cette dernière décennie. Plutôt que de réaliser des ajustements statistiques sur les observations, la méthode s'attache à modéliser les processus hydrologiques pour générer des chroniques de débits simulés d'où sont tirées des grandeurs statistiques liées à la ressource en eau : caractéristiques d'étiage, de bilan et de saisonnalité. La modélisation hydrologique utilisée permet de simuler des chroniques de débits journaliers et fournit avec une seule cartographie de ses paramètres, l'ensemble des caractéristiques hydrologiques pour les sites non jaugés. Cet article présente l'approche appliquée au territoire national français et les résultats obtenus intégrés dans une base de données accessible pour les services de l'État via une interface Web.

\section{Les données}

\subsection{Les données hydrométéorologiques observées}

Nous utilisons une information hydrométéorologique la plus exhaustive possible, disponible sur les bases de données nationales: données météorologiques issues de la réanalyse SAFRAN de Météo-France, données hydrologiques issues de la banque Hydro (http://www.hydro.eaufrance.fr/). Les précipitations solides et liquides ainsi que les températures de SAFRAN sont utilisées. L'ETP moyenne utilisée est calculée à partir de la formule établie par Oudin et al. (2005). Cette ETP dépend uniquement de la température de l'air et du rayonnement extraterrestre. L'ensemble de ces informations est disponible sur le territoire national, à la maille de 8 par $8 \mathrm{~km}$ sur la période 1958-2018. Les données hydrométriques se présentent sous la forme de chroniques de débits journaliers. Les durées des chroniques sont variables suivant les bassins mais couvrent au minimum 25 ans de données sur la période du 01/01/1970 au 31/12/2018.

Le calage de la méthode porte sur 690 bassins versants du territoire français couvrant une diversité de régimes hydrologiques et conditions hydro-climatiques. Ces bassins ont été sélectionnés en fonction de la qualité de la mesure, de la longueur de leur chronique, de la continuité de leur disponibilité sur la période étudiée et de leur fonctionnement dit «naturel» ou «non influencé».

\subsection{Les indicateurs sur la ressource en eau dérivés des chroniques hydrométéorologiques}

L'objectif du travail présenté est la détermination d'indices hydrologiques dérivés des chroniques de débits journaliers simulés en contexte non jaugé, permettant de qualifier efficacement les régimes d'étiage. Ces indices décrivent de manière exhaustive les étiages. Ils sont calculés à différents pas de temps, et caractérisent différentes sévérités de l'évènement. Les indices préconisés par la législation française pour le calcul des débits réservés à l'aval des ouvrages hydrauliques ou pour la limitation des rejets sont également calculés. Enfin, les statistiques retenues présentent aussi des indices marqueurs de la saisonnalité des débits et du bilan annuel. Ces indices calculés sont répertoriés dans le tableau 1 dans les lignes grisées et notés (IH). Parmi ces indices, le QMNA, principalement utilisé en France, correspond au débit mensuel minimal annuel et le VCNd est le Volume Consécutif Minimal pour d jours. Le QMNA est très proche du VCN30, il lui est privilégié dans la législation française. Cependant, si l'étiage ne correspond pas à un mois calendaire, et qu'il est superposé sur deux mois, le QMNA aura tendance à surestimer la valeur représentative de l'étiage, ce qui n'est pas le cas avec le VCN30. Des caractéristiques de la courbe des débits classés qui est une signature hydrologique importante d'un bassin versant, ont aussi été sélectionnées. Elles renseignent sur la fréquence de dépassement associée à une valeur de débit. Ainsi le $\mathrm{DC}_{50}$ correspond à un débit dépassé environ 183 jours par an. Il indique en outre la séparation entre les conditions de basses eaux et de hautes eaux de l'écoulement du cours d'eau. Le $\mathrm{DC}_{75}$ correspond à un débit non dépassé environ 91 jours 
Tableau 1. Description des indices hydrologiques $(\mathrm{IH})$ utilisés pour le calage et la régionalisation de la méthode (indiqués dans les lignes grisées du tableau) et description d'autres indicateurs hydrologiques déduits des simulations régionales (en italique dans le tableau).

\begin{tabular}{|c|c|}
\hline Nom & Description des Indices Hydrologiques (IH) \\
\hline & Étiage \\
\hline $\mathrm{QMNA}_{2}, \mathrm{QMNA}_{5}, \mathrm{QMNA}_{10}$ & $\begin{array}{l}\text { Débit mensuel minimal annuel de période de retour } 2,5 \text { et } 10 \text { ans. Ajusté à la loi log-normale. [m³ } / \mathrm{s} \\
\text { ou } 1 / \mathrm{s} \text { ] }\end{array}$ \\
\hline $\begin{array}{l}\mathrm{VCN} 10_{2}, \mathrm{VCN} 10_{5} \\
\text { VCN10 }\end{array}$ & $\begin{array}{l}\text { Volume Consécutif minimal pour } 10 \text { jours de période de retour } 2,5 \text { et } 10 \text { ans Ajusté à la loi } \log \text { - } \\
\text { normale. }\left[\mathrm{m}^{3} / \mathrm{s} \text { ou } 1 / \mathrm{s}\right] \text {. }\end{array}$ \\
\hline $\mathrm{VCN} 3_{2}, \mathrm{VCN} 3_{5}, \mathrm{VCN} 3_{10}$ & $\begin{array}{l}\text { Volume Consécutif minimal pour } 3 \text { jours de période de retour } 2,5 \text { et } 10 \text { ans. Ajusté à la loi log- } \\
\text { normale. }[\mathrm{m} 3 / \mathrm{s} \text { ou } 1 / \mathrm{s}] \text {. }\end{array}$ \\
\hline \multirow[t]{2}{*}{$\begin{array}{l}\text { Quantiles des débits classés: } \\
\mathrm{DC}_{95}, \mathrm{DC}_{90} \mathrm{DC}_{80} \mathrm{DC}_{70} \mathrm{DC}_{50}\end{array}$} & $\begin{array}{l}\text { Quantiles de débits issus de la courbe des débits classés calculée sur l'échantillon entier (Hughes et } \\
\text { Smakhtin, 1996); [m3/s ou } 1 / \mathrm{s}] \text {. Certaines valeurs de débits sont indiquées: DCx, débit classé de } \\
\text { quantile } \mathrm{x} \% \text {, il correspond à un débit égalé ou dépassé environ } \mathrm{x} \% * 365 \text { jour par an. }\end{array}$ \\
\hline & Saisonnalité \\
\hline \multirow[t]{2}{*}{$\mathrm{QM}_{1} \mathrm{à} \mathrm{QM}_{12}$} & Débits mensuels moyen $[\mathrm{m} 3 / \mathrm{s}$ ou $1 / \mathrm{s}]$ \\
\hline & Bilan \\
\hline QA & Ecoulement moyen annuel ou module [m3/s ou 1/s] \\
\hline$Q a$ & Débits annuels moyens $[\mathrm{m} 3 / \mathrm{s}$ ou $\mathrm{l} / \mathrm{s}]$ \\
\hline Coefficient de ruissellement & Rapport : $Q A / P A[-]$ \\
\hline Fraction Apport Neigeux & Fraction d'apport neigeux : Pluie solide/Pluie liquide [-] \\
\hline Indice d'aridité & Rapport : ETPA/PA [-]; avec PA: Précipitation annuelle moyenne et ETPA: ETP annuelle moyenne \\
\hline BFI (Base Flow Index) & $\begin{array}{l}\text { Il décrit l'écoulement de base. La valeur du BFI est calculée selon la méthode de " smoothed } \\
\text { minima technique » (package R Ifstat) [-] }\end{array}$ \\
\hline Ratio $D C_{90} / D C_{50}$ & $\begin{array}{l}\text { Définit l'inertie de l'étiage : rapport des quantiles } D C_{90} \text { et } D C_{50} \text { de la courbes des débits classés } \\
{[-]}\end{array}$ \\
\hline
\end{tabular}

par an, indice d'étiage peu sévère mais qui permet de caractériser les rivières possédant des étiages importants. Et le $\mathrm{DC}_{95}$ correspond à un débit non dépassé 18 jours par an. Tous ces indices ont été calculés sur les observations et utilisés pour le calage du modèle hydrologique. A ces variables, différents indicateurs hydrologiques ont été ajoutés. Ils n'ont été calculés que pour renseigner la base de données et n'ont pas servi au calage de la méthode. Ils sont renseignés en italique dans le tableau 1, dans les lignes non grisées.

\section{Méthodologie de la mise en œuvre de la méthode LoiEau}

\subsection{Le modèle pluie-débit}

La structure du modèle hydrologique est globale et elle a été optimisée. Le modèle est contraint par seulement deux paramètres (Garcia, 2016) afin qu'il soit facilement régionalisable pour être utilisé sur des bassins non jaugés. Ce modèle pluie-débit journalier de la famille des modèles GR (Michel, 1983) s'est largement inspiré d'une structure mensuelle à deux paramètres (Folton et Arnaud, 2012). La structure finale du modèle dénommée GRLoieau2J est composée de trois réservoirs (Fig. 1):

- un réservoir de production de capacité maximale A (mm) est alimenté par des données journalières de pluie liquide, Pliq( $(\mathrm{t})$, et d'évapotranspiration potentielle, $\mathrm{E}(\mathrm{t})$, calculées à l'échelle du bassin versant. Ce réservoir assure le bilan en eau de surface et calcule la part de la pluie qui contribue à

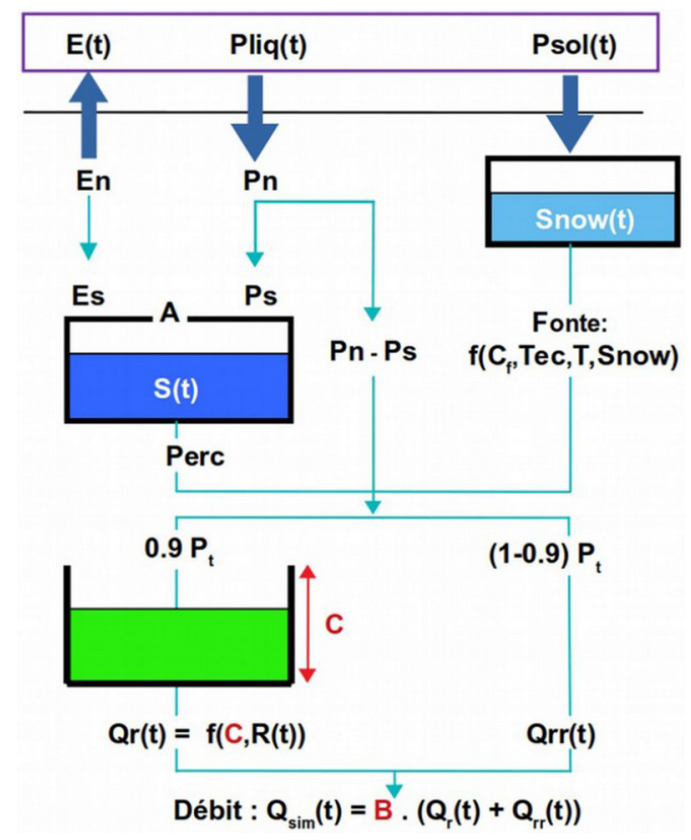

Fig. 1. Modèle hydrologique $\mathrm{GR}_{\text {Loieau2J. }}$.

l'écoulement $(\mathrm{Pt}=\mathrm{Pn}-\mathrm{Ps})$ en fonction de son niveau de remplissage $\mathrm{S}(\mathrm{t})$. Cette structure est similaire au module de production du modèle hydrologique GR4J (Perrin et al., 2003). 
- un réservoir stocke la pluie solide, Psol(t), calculée à l'échelle du bassin versant pour déterminer un stock de neige, Snow(t). La loi de vidange de ce réservoir est inspirée de la formule degré-jour couramment utilisée en hydrologie opérationnelle. Cette loi de vidange possède deux paramètres, un coefficient de vidange $\left(\mathrm{C}_{\mathrm{f}}\right)$ et une température de fonte $(\mathrm{Tec})$. Ces deux paramètres sont régionalisés; le coefficient de fonte est régionalisé par massif montagneux et la température de fonte est imposée à $-1{ }^{\circ} \mathrm{C}$ (Folton et Coulier, 2011). Le processus de fonte s'active uniquement lorsque la température journalière est supérieure à la température de fonte fixée. La pluie contribuant à l'écoulement est la somme de la fonte, de la pluie non routée vers le réservoir $\mathrm{S}$, et d'une percolation issue de $\mathrm{S}$.

- un réservoir de routage de capacité maximale « $\mathrm{C} »$, permet de retarder $90 \%$ de l'écoulement, suivant une loi de vidange dépendant du niveau de remplissage $\mathrm{R}(\mathrm{t})$. Les $10 \%$ restants transitent directement vers la rivière. Le débit total dans la rivière est constitué de la sortie du réservoir de routage et du débit écoulé directement, corrigé par le paramètre $\langle\mathrm{B} » d u$ modèle. L'autre paramètre du modèle noté $« \mathrm{C}$ » est la capacité à un jour du réservoir de routage exprimée en $\mathrm{mm}$. La figure 1 présente schématiquement la version de la modélisation retenue.

Au niveau du réservoir de production, un bilan hydrique est effectué ainsi qu'une percolation (Perc), mais on ne tient pas compte d'infiltrations ou d'échanges souterrains potentiels, ni de l'évapotranspiration réelle du bassin. La capacité maximale notée A de ce réservoir n'est pas optimisée mais a été imposée sur toute la France où elle varie entre 200 et $250 \mathrm{~mm}$ en fonction de l'hydrogéologie. Le paramètre $\mathbf{B}$ permet de prendre en compte indirectement les échanges souterrains non modélisés par la fonction de transfert et les incertitudes liées à la production, en corrigeant le débit sortant. Le bilan en eau est assuré par cette correction. Ce paramètre rend compte de tous ces échanges non modélisés par un apport $(\mathbf{B}>1)$ ou une perte $(\mathbf{B}<1)$ autre que la pluie ou l'évapotranspiration. Le paramètre $\mathbf{C}$ est exprimé en $\mathrm{mm}$. Il représente la capacité maximale du réservoir de transfert. Il intervient dans l'équation de vidange du réservoir de transfert et permet au modèle de reproduire la variabilité temporelle du débit journalier. Plus la valeur de $\mathbf{C}$ est petite, plus la variabilité du débit augmente et plus la valeur de $\mathbf{C}$ est grande, plus le pic de crue journalière est atténué et la décrue est lente. Il génère donc une dynamique à l'écoulement.

\subsection{La fonction objectif}

Le calage du modèle (paramètres $\mathrm{B}$ et $\mathrm{C}$ ) a consisté à trouver une paramétrisation du modèle hydrologique qui permet de s'approcher au mieux de la restitution de l'ensemble des indices statistiques de calage observés (étiage, saisonnalité et écoulement moyen annuel). Garcia et al. (2017) a montré que la fonction objectif définie par la moyenne entre le KGE, critère de Kling et Gupta, appliqué au débit journalier et le KGE appliqué à l'inverse du débit est la plus appropriée pour remplir toutes ces conditions d'évaluation. L'algorithme d'optimisation utilisé est celui du package «airGR». Il permet d'identifier les paramètres en maximisant le critère de calage en deux temps : l'exploration exhaustive de l'espace des paramètres puis la recherche locale de l'optimum.

\subsection{La régionalisation du modèle}

Le calage des paramètres du modèle hydrologique n'est possible que si le bassin versant étudié possède des mesures de débit à son exutoire. En l'absence de débits mesurés, le calage ne peut être effectué sur le bassin et la connaissance de ces débits repose sur un transfert de l'information hydrologique disponible sur d'autres bassins. La régionalisation du modèle hydrologique consiste donc à transférer les paramètres du modèle calé sur les bassins jaugés (dits aussi donneurs) vers les bassins voisins non jaugés (dits aussi receveurs). La régionalisation choisie est basée sur la proximité spatiale (dite aussi proximité géographique). En France, cette méthode utilisée avec les modèles pluie-débit fournit une solution de régionalisation performante (Oudin et al., 2008). Pour déterminer la proximité spatiale d'un bassin receveur, la pondération par l'inverse de la distance a été retenue. Sa formule est donnée par l'équation (1):

$$
Z_{j}=\frac{\sum_{i=1}^{n \neq j} \frac{1}{d_{i}^{p}} Z_{i}}{\sum_{i=1}^{n \neq j} \frac{1}{d_{i}^{p}}}
$$

où $n$ est le nombre de voisins, $p$ est la puissance, $d$ est la distance entre deux bassins (distance entre les centroïdes) et $Z$ est un paramètre du modèle pluie-débit à interpoler pour le bassin receveur $j$ à partir des bassins donneurs $i$ sélectionnés.

La formulation n'autorise pas un bassin à faire partie de son propre voisinage, c'est à dire que $i$ et $j$ ne peuvent pas être égaux. Cela signifie que pour estimer la valeur de $Z$ sur un site donneur, on n'utilise pas la valeur de $Z$ calée et connue en ce site. On procède donc à une sorte de validation croisée parmi les sites donneurs. Le fait de connaître la valeur optimale du paramètre peut donc permettre de calculer une erreur d'estimation et donc d'optimiser la structure du voisinage, c'est à dire les paramètres $n$ et $p$. La configuration optimale a été estimée en optimisant le RMSE calculé entre la distribution de chaque paramètre calé et chaque paramètre régionalisé. Cela a conduit à retenir, une puissance $p$ de 2, et un nombre $n$ de voisins de 11. Par ailleurs un traitement des bassins versants dits «horsains » a été appliqué par l'utilisation de méthodes itératives afin de limiter leur impact.

\subsection{Le calcul des intervalles de confiance}

L'étude des incertitudes est relativement complexe dans ce genre de méthode qui combine plusieurs étapes de modélisation (calage et régionalisation) et multiplie de fait, les facteurs d'incertitude. Cependant, un travail a permis une estimation de l'incertitude liée à la mise en œuvre de la méthode. Ces incertitudes sont réalisées en ré-échantillonnant les pluies, les ETP et les chroniques de débits afin de permettre la prise en compte de l'incertitude sur les données d'entrées. L'approche 
consiste à générer 100 échantillons de 25 ans de données, construits par tirage aléatoire dans l'ensemble des années disponibles de la période de 1970 à 2018 . Le modèle est calé sur les 100 échantillons de 25 ans, pour chaque bassin versant jaugé (les périodes de 25 ans de données ré-échantillonnées sont communes à tous les bassins). Nous obtenons 100 jeux de paramètres pour chaque bassin jaugé, et nous simulons ensuite 100 chroniques sur 48 ans (de 1970 à 2018) à partir desquelles sont calculées les statistiques de bilan, de saisonnalité et d'étiage à différents pas de temps. On obtient ainsi 100 valeurs pour les indices hydrologiques (IH). Pour chacun d'entre eux, une mise en fréquence permet de déterminer la médiane des 100 valeurs obtenues ainsi que les quantiles $5 \%\left(\mathrm{IH}_{5 \%}\right)$ et $95 \%$ $\left(\mathrm{IH}_{95 \%}\right)$. Les intervalles de confiance sont évalués par le calcul de l'amplitude $\mathrm{A}_{90 \%}$ égale à l'écart entre ces deux quantiles, mais peut aussi être exprimé en relatif par le calcul de l'amplitude relative suivante $\mathrm{Ar}_{90 \%}$ :

$$
A r_{90 \%}=\frac{\left(I H_{95 \%}-I H_{5 \%}\right)}{I H_{50 \%}}
$$

où $\mathrm{IH}_{5 \%}, \mathrm{IH}_{95 \%}$ et $\mathrm{IH}_{50 \%}$ représentent respectivement les quantiles $5 \%, 95 \%$ et $50 \%$ de la distribution des indices hydrologiques.

Les incertitudes régionales sont ensuite abordées en mettant en œuvre 100 fois la régionalisation, à partir des 100 jeux de paramètres issus des calages réalisés sur les données d'entrées ré-échantillonnées. Cela consiste à transférer les 100 jeux de paramètres obtenus sur les bassins jaugés (donneurs) aux bassins non jaugés (receveurs). Cette opération demande que l'application de la régionalisation soit suffisamment «automatisable» pour être appliquée un grand nombre de fois. Au final, nous obtenons, sur chaque bassin non jaugé, 100 chroniques de débits simulés à partir desquelles nous calculons les indices hydrologiques. Comme précédemment, l'amplitude relative à $90 \%\left(\mathrm{Ar}_{90 \%}\right)$ propre à chaque indice hydrologique est calculée et fournit ainsi une estimation de l'incertitude pour chaque indice sur les bassins non jaugés.

\section{Les performances de la méthode}

Afin d'évaluer les performances de la méthode dans la restitution des indices statistiques simulés, une comparaison est effectuée avec une approche plus couramment utilisée en hydrologie, la régionalisation directe d'un quantile. Cette comparaison est menée en validation croisée sur notre échantillon de 690 stations. La procédure est réalisée autant de fois que de stations présentes dans l'échantillon, et le processus d'exclusion est mené de telle sorte que chaque station est exclue une seule fois. L'échantillon amputé d'une station sert à régionaliser les modèles et la validation est effectuée sur la station exclue. La restitution de trois indices en sites non jaugés est testée: QA, $\mathrm{QMNA}_{5}$ et $\mathrm{VCN}_{5}$. L'approche directe consiste à établir des liens explicites entre les indices de débits et des caractéristiques descriptives des bassins versants. Ces caractéristiques comprennent des descripteurs climatiques, morphologiques, géologiques, pédologiques et d'occupation du sol. La recherche de la meilleure régression linéaire multiple a été menée, pour chacun des trois indices, avec une interpolation spatiale des résidus à la

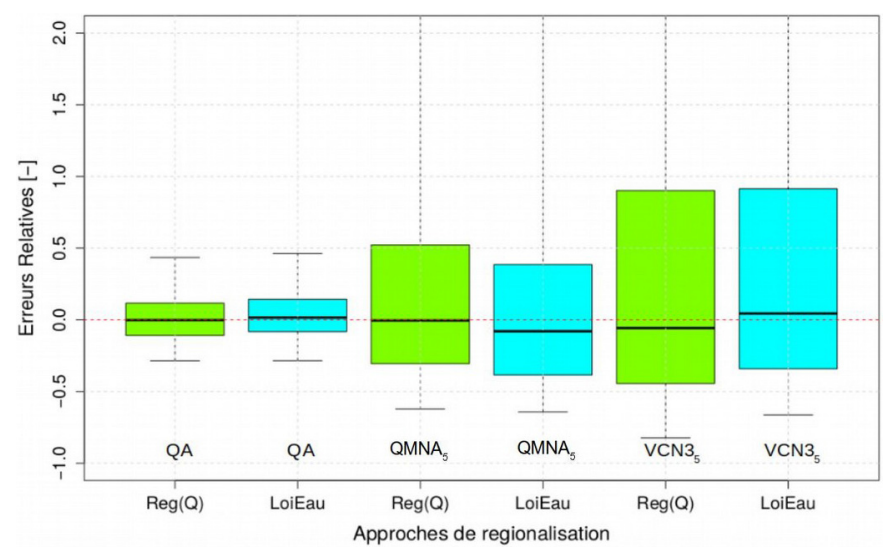

Fig. 2. Distributions des performances pour la simulation des trois indices hydrologiques $\left(\mathrm{QA}, \mathrm{QMNA}_{5}\right.$ et $\left.\mathrm{VCN}_{5}\right)$ en contexte non jaugé selon les deux approches ; approche $\operatorname{Reg}(\mathrm{Q})$ en vert : régression multiple du quantile avec interpolation des résidus; approche LoiEau en bleu: modèle hydrologique régionalisé.

régression. Au final, les modèles de régression ont fait appel essentiellement à des descripteurs climatiques et morphologiques. Ainsi, la pluviométrie annuelle et l'altitude moyenne des bassins versants expliquent la variabilité de l'écoulement annuel moyen (QA) tandis que la pente moyenne du bassin, la densité de drainage et la pluviométrie annuelle ont un rôle plus important pour les deux variables de l'étiage.

La figure 2 compare les distributions d'erreurs relatives obtenues pour les trois indices statistiques et selon les deux approches sur l'échantillon total : la régionalisation des indices par régression globale avec interpolation des résidus, notée $\operatorname{Reg}(\mathrm{Q})$ et l'estimation par le modèle hydrologique régionalisé, notée LoiEau. Globalement, les distributions des erreurs pour les deux approches sont relativement proches, mais la méthode LoiEau présente l'avantage d'être globale et non limitée à une seule variable hydrologique.

\section{Les résultats}

\subsection{La base de données Web LoiEau}

La base de données LoiEau (BD LoiEau) est donc le résultat de l'application de la méthode LoiEau à l'exutoire de bassins non instrumentés. Les bassins versants topographiques ont été calculés à partir de directions d'écoulement disponibles à un pas de $50 \mathrm{~m}$. Cette base des directions d'écoulement fournie par le SCHAPI, peut être considérée comme une base de référence nationale pour la délimitation des bassins versants topographiques. À chaque exutoire est associé son bassin versant ainsi qu'un tronçon hydraulique délimité par l'exutoire en question et l'exutoire immédiatement à l'amont. La méthode régionale a été mise en œuvre sur les 130000 bassins de cette base, ayant une superficie comprise entre 5 et $8000 \mathrm{~km}^{2}$. Nous obtenons des chroniques hydrologiques simulées de 1970 à 2018, au pas de temps journalier à l'exutoire de chaque bassin. À partir de ces débits simulés, toutes les statistiques définies dans le tableau 1 sont calculées sur une période identique et sont associées à des intervalles de confiance comme indiqué au paragraphe III.4. 


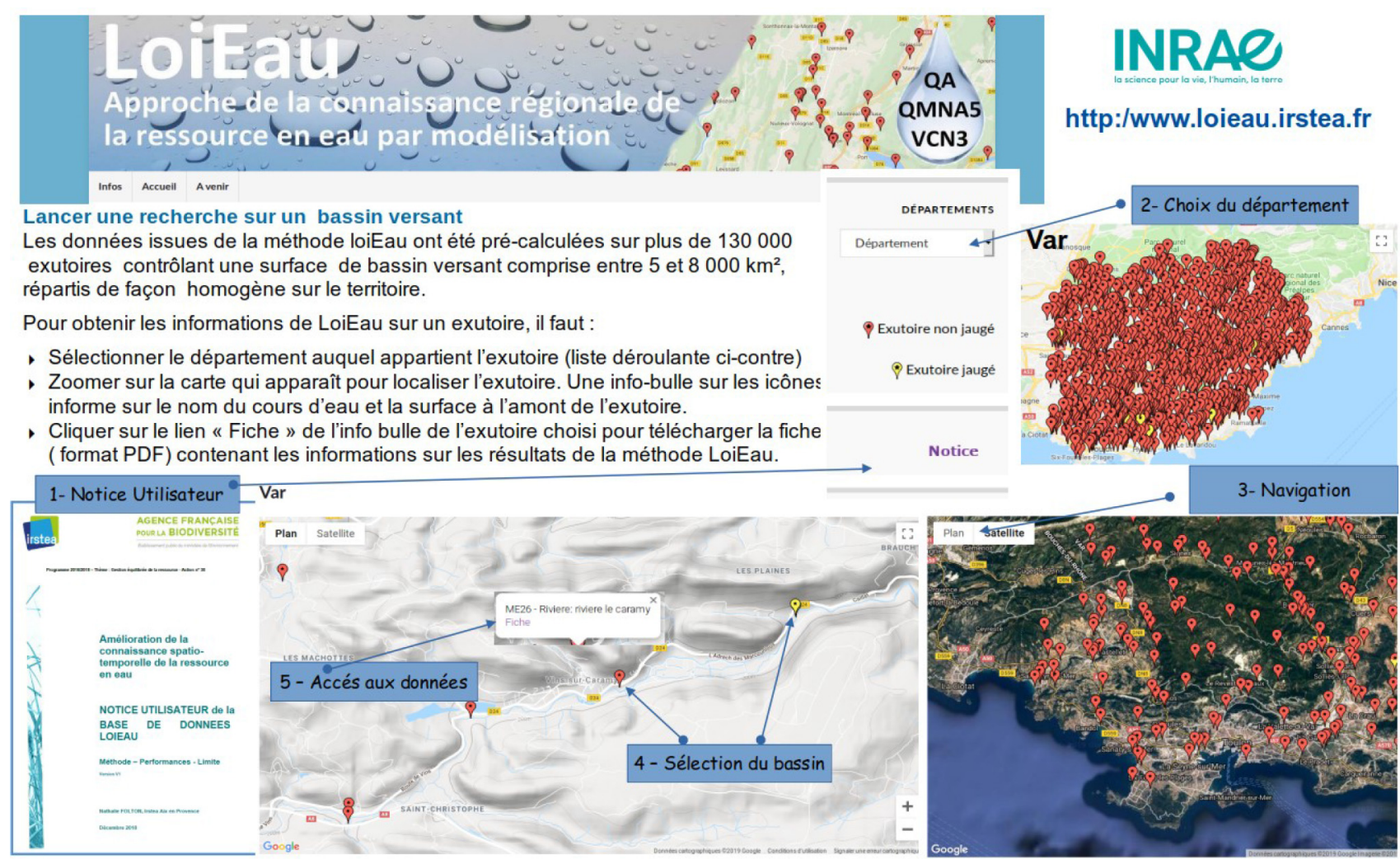

Fig. 3. Portail de l'application Web LoiEau.

Une application Web (https://loieau.irstea.fr/) gère la base de données. L'application a été développée sous WordPress, avec le langage de scripts PHP. Elle est reliée à Google Maps afin de bénéficier d'une représentation cartographique des exutoires des bassins versants offrant des possibilités de zoom et de recherche (Fig. 3).

Les bassins modélisés sont accessibles par sélection d'un département dans le menu principal. Une carte apparaît avec la localisation des bassins modélisés. Les exutoires sont représentés par des symboles, de couleur jaune pour les bassins jaugés et de couleur rouge pour les bassins non jaugés. Un simple clic sur ce symbole permet d'accéder à l'info-bulle, laquelle renseigne le nom du cours d'eau et la surface drainée en amont de ce point. L'accès aux données modélisées sur le bassin choisi est réalisé via le lien dénommé «Fiche» de l'infobulle. Les données sont rassemblées dans un fichier au format «pdf», lequel contient plusieurs pages. La figure 4 présente la fiche de synthèse d'un bassin modélisé sur le cours d'eau «Le Caramy » dans le département du Var.

Sur la première page, un plan de situation permet de localiser l'exutoire du bassin versant et son tracé issu d'un modèle numérique de terrain. Les caractéristiques du bilan, de la saisonnalité et de l'étiage, sont regroupées dans un tableau et calculées sur la totalité de la période ainsi que sur trois autres périodes de 30 ans, décalées d'une décade (1970-1990, 1980-2000, 1990-2015). Les intervalles de confiance sont indiqués pour chaque indice hydrologique. Les pages suivantes contiennent les débits annuels, mensuels et journaliers simulés, liés au bilan, à la saisonnalité et à l'étiage du bassin versant.

\subsection{Les limites d'utilisation}

La méthode est calée sur des bassins versants jugés «non influencés », c'est-à-dire correspondant à des fonctionnements dits «naturels». C'est aussi une méthode régionalisée, ce qui implique parfois des simplifications nécessaires pour transposer la méthode. La variabilité spatiale des processus peut alors être plus ou moins bien restituée sur des bassins versants non-jaugés sur lesquels on ne dispose pas d'information suffisante pour prendre en compte la réalité des phénomènes. Certains bassins peuvent donc présenter des fonctionnements pouvant être contraints par différentes configurations mettant en défaut la méthode. Des codes de confiance en la méthode ont donc été calculés sur chaque bassin versant pour donner une indication sur les particularités présentes sur le bassin versant considéré. Cependant, ils ne prévoient en aucun cas une correction à apporter sur les quantiles de débit ou caractéristiques d'étiage. Ils sont proposés pour signaler les zones particulières pour lesquelles les débits simulés par la méthode LoiEau peuvent être remis en cause. Il est important que l'utilisateur soit sensibilisé par l'alerte donnée par cet indice de confiance dans le cas où les spécificités locales rendent les résultats inappropriés. Les spécifités retenues comprennent les influences liées à la présence d'ouvrages hydrauliques, les influences des prélèvements en eaux superficielles, les influences liées à la présence de zones karstiques dans le bassin, de zones plates ou d'apports externes au territoire français dans le cas de bassins frontaliers. Ces influences sont quantifiées individuellement sur chaque bassin et sont associées à un indice de confiance allant de « 1 » (bassin 


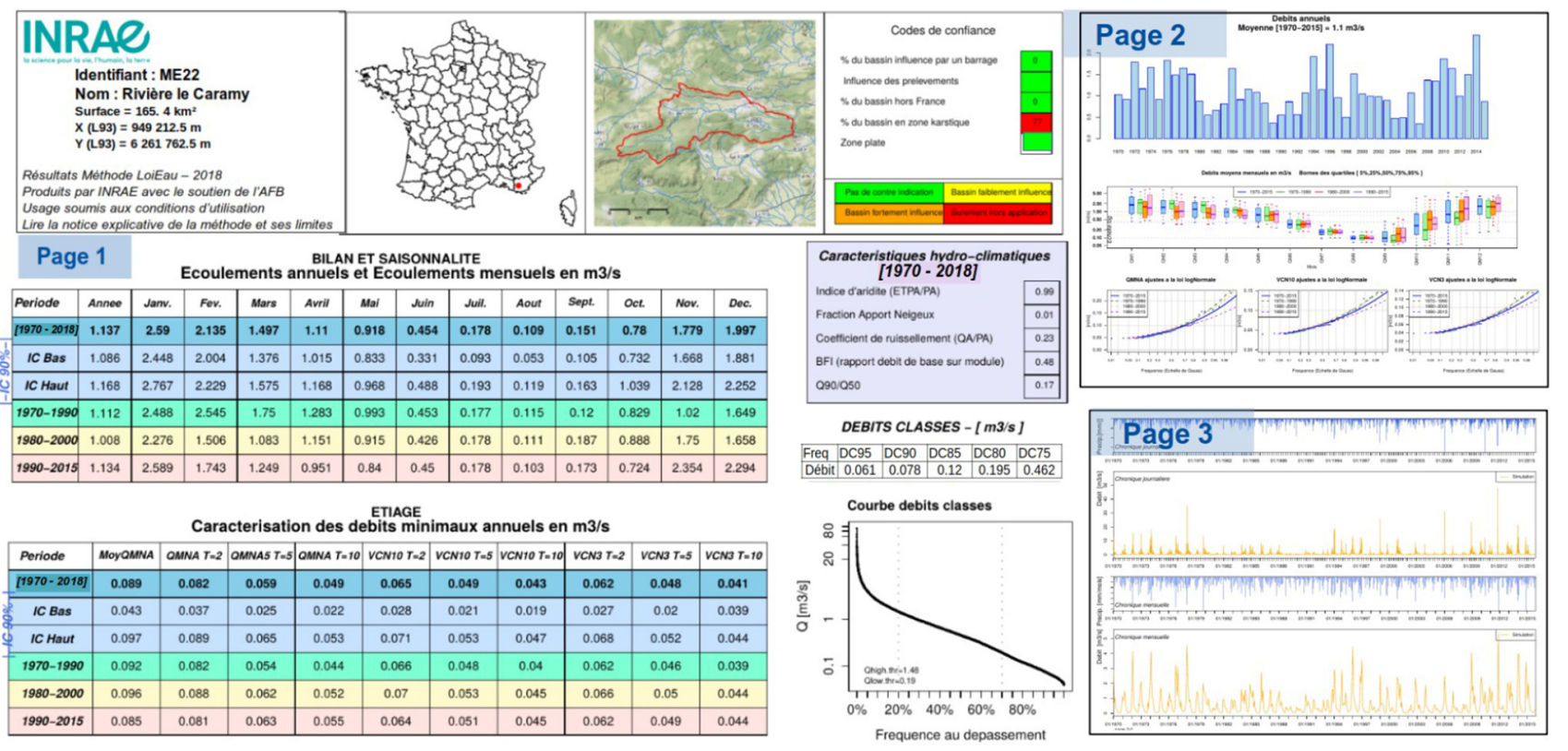

Fig. 4. Fiche «PDF» des résultats de la méthode sur un bassin versant non jaugé. La page 1 situe le bassin et indique les principaux indices hydrologiques calculés, la page 2 présente les débits annuels, les débits saisonniers et les ajustements des différents débits d'étiage simulés, la page 3 présente les tracés des débits journaliers et mensuels simulés.

(a)

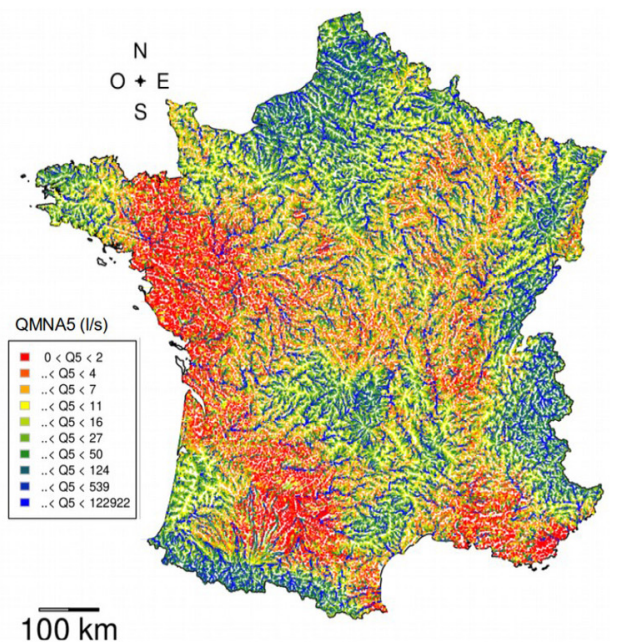

(b)

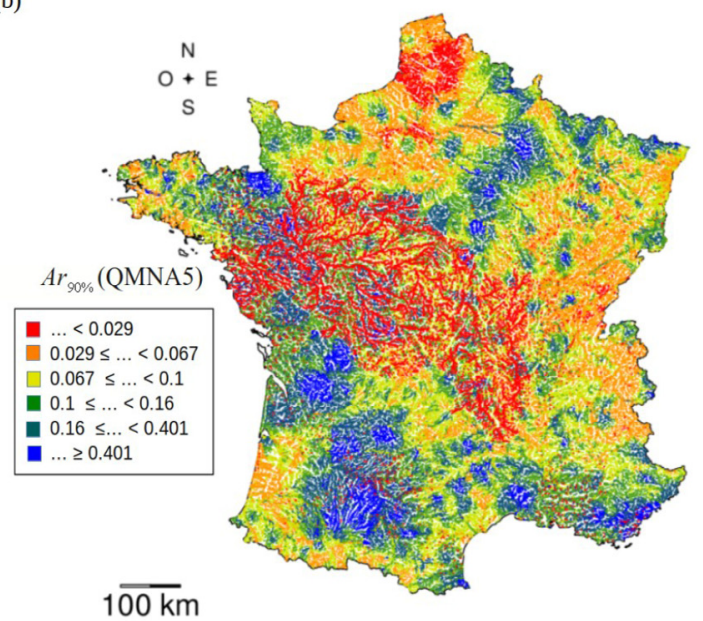

Fig. 5. Cartographie du QMNA5 exprimé en $1 / \mathrm{s}$ (a) et de son amplitude relative $A r_{90 \%}$ (b), calculés sur l'ensemble des points du réseau hydrographique. Seuls les points drainant une surface supérieure à $25 \mathrm{~km}^{2}$ sont représentés afin de ne pas surcharger les cartes.

probablement en dehors du champ d'application de la méthode) à « 4 »(pas de contre-indication). La quantification de chaque influence est détaillée dans la notice d'accompagnement de la méthode et de ses résultats, téléchargeable sur le portail de la base.

\subsection{Les cartographies des indices hydrologiques et leurs incertitudes}

A partir des indices hydrologiques calculés sur l'ensemble des bassins non jaugés de la base, nous pouvons représenter une cartographie de chaque indice et de leurs intervalles de confiance. À titre d'exemple la figure 5 représente la cartographie du QMNA $\mathrm{Q}_{5}$ exprimée en 1/s (à gauche) et son amplitude relative associée $\operatorname{Ar}(90 \%)$ (à droite). Tous les indices hydrologiques calculés par la méthode peuvent faire l'objet d'une cartographie et être associés à son incertitude. Ce travail de cartographie présente l'avantage de représenter de façon synthétique, les données sur les ressources en eau à l'échelle du territoire métropolitain français. Ainsi, la cartographie de la figure 5a permet de visualiser à l'échelle du territoire les écoulements mensuels liés à l'étiage quinquennal et de localiser les plus fortes incertitudes de la méthode sur la figure 5b. Celles-ci sont localisées dans l'ensemble du bassin amont de la Garonne, à l'est de la région Seine-Normandie, en région Poitou Charente et en région méditerranéenne pour le $\mathrm{QMNA}_{5}$. 


\section{Discussion et conclusions}

La méthode LoiEau permet d'estimer en tout point d'un cours d'eau, des indices d'étiage, de saisonnalité ou de bilan, sur des intervalles de temps journaliers ou supérieurs, grâce à un modèle pluie-débit journalier régionalisé. L'objectif est d'utiliser un modèle simple et suffisamment flexible pour être applicable à une large variété de fonctionnements hydrologiques et pour être utilisé sous différentes conditions climatiques et environnementales. Le contrôle de la qualité de la méthode par validation croisée apporte la confirmation que celle-ci fournit une information pertinente non pas uniquement sur les seuls bassins utilisés pour la régionalisation mais sur l'ensemble du territoire étudié.

La diversité hydroclimatique de l'échantillon de bassins versants est nécessaire pour garantir une robustesse à la méthode. Cette diversité se retrouve aussi dans le comportement des bassins versants vis à vis des caractéristiques d'étiage, avec des bassins pour lesquels l'étiage est particulièrement soutenu par des apports souterrains. Ces bassins à comportement uniforme montrent les performances de simulation les plus faibles par rapport aux observations. Une perspective envisageable est d'améliorer leur modélisation avec une prise en compte des écoulements souterrains. Des travaux de thèse ont récemment débuté sur cette problématique.

Pour aider les gestionnaires à avoir une meilleure idée du niveau de confiance à accorder aux estimations produites, une méthode simple de quantification des incertitudes liées au calage de la méthode a été introduite et restitue systématiquement pour chaque valeur d'indice hydrologique une estimation de l'incertitude par un intervalle de confiance. Des codes de confiance en la méthode ont été aussi introduits afin d'alerter les utilisateurs sur les zones particulières pour lesquelles les hypothèses associées à la méthode peuvent être remises en cause.

La cartographie du QA et du QMNA5 décrite dans cet article constitue une des trois estimations intégrées dans la cartographie de référence de ces deux variables diffusée par l'Agence Française pour la Biodiversité (AFB) sur le portail EauFrance (https://www.eaufrance.fr/). Cette cartographie, disponible uniquement pour ces deux variables permet, par une combinaison des estimations de trois méthodes différentes de prendre en compte les qualités des trois méthodes appliquées pour améliorer l'estimation finale des débits. Cette estimation finale est vue comme une estimation complémentaire pour ces deux variables et les résultats sont disponibles dans les fiches de la base LoiEau.

Cette approche par modélisation hydrologique, peut aussi permettre la prise en compte d'hypothèses de scénarios de changements climatiques ou d'occupation du sol. Ainsi différents scénarios climatiques continus tels que ceux fournis par le modèle CNRM-CM3 développé par le CERFACS et le CNRM de Météo-France, ont pu être testés afin de dégager les conséquences de l'évolution climatique sur les écoulements d'étiage des bassins versants français (Folton et al., 2012).

Remerciements. Nous remercions l'Agence Française pour la Biodiversité (AFB) pour son soutien financier ainsi que sa chargée de mission Claire Magand pour ses conseils avisés durant le suivi du projet. Nous remercions aussi Météo-France pour la mise à disposition des données SAFRAN pour la mise en œuvre de ce travail de recherche autour de la modélisation hydrologique.

\section{Références}

Blöschl G, Sivapalan M, Wagener T, Viglione A, Savenije H. 2013. Runoff prediction in ungauged basins: Synthesis across processes, places and scales. Cambridge University Press, $490 \mathrm{p}$.

Folton N, Arnaud P. 2012. Régionalisation d'une modélisation hydrologique pour la cartographie des débits d'étiage. In: $9^{e}$ Congrés International GRUTTEE, Aix-en-Provence, France.

Folton N, Coulier P. 2011. La méthode LoiEau. Reformulation du module «Neige », Rapport d'étude ONEMA/IRSTEA, 45 p.

Folton N, Cantet P, Arnaud P, Fouchier C, Turquois L. 2012. Prise en compte de scénarios de changement climatique dans des méthodes de cartographie de l'aléa hydrologique-Application aux pluies intenses et aux débits mensuels et annuels. In: Les changements globaux enjeux et défis. Toulouse, France.

Garcia F. 2016. Amélioration d'une modélisation hydrologique régionalisée pour estimer les statistiques d'étiage. Thèse de doctorat, Irstea UPMC Paris VI, $250 \mathrm{p}$.

Garcia F, Folton N, Oudin L. 2017. Which objective function to calibrate rainfall-runoff models for low-flow index simulations? Hydrol Sci J 62(7): 1-18. https://doi.org/10.1080/ 02626667.2017.1308511.

Laaha G, Skoien JO, Bloschl G. 2013a. Spatial prediction on river networks: Comparison of top-kriging with regional regression. Hydrol Process 28(2): 315-324.

Laaha G, Demuth S, Hisdal H, et al. 2013b. Prediction of low flows in ungauged basins. In: Blöschl G, Sivapalan M, Wagener T, Viglione A, Savenije H, eds. Runoff prediction in ungauged basins: Synthesis across processes, places and scales. Cambridge University Press, $490 \mathrm{p}$.

Michel C. 1983. Que peut-on faire en hydrologie avec un modèle à un paramètre? La Houille Blanche 1: 39-44.

Oudin L, Michel C, Anctil P. 2005. Which potential evapotranspiration input for a lumped rainfall-model? Part 2-Towards a simple and efficient potential evapotranspiration model for rainfall-runoff modelling. J Hydrol 303(1-4): 290-306.

Oudin L, Andréassian V, Perrin C, Michel C, Le Moine N. 2008. Spatial proximity, physical similarity, regression and ungaged catchments: A comparison of regionalization approaches based on French catchments. Water Resour Res 44.

Perrin C, Michel C, Andreassian V. 2003. Improvement of a parsimonious model for streamflow simulation. J Hydrol 279(1-4): 275-289.

Citation de l'article : Folton N, Arnaud P. 2020. Indicateurs sur la ressource en eau estimés par une modélisation pluie-débit régionalisée : la base de données Web LoiEau. La Houille Blanche : 22-29 